\title{
Nested Variant of Urothelial Carcinoma: A Clinicopathologic and Immunohistochemical Study of 12 Cases
}

Oscar Lin, M.D., Marina Cardillo, M.D., Guido Dalbagni, M.D., Irina Linkov, Brian Hutchinson, Victor E. Reuter, M.D.

Department of Pathology (OL, IL, BH, VER) and Department of Urology (GD), Memorial Sloan-Kettering Cancer Center, New York and Department of Pathology, Jacobi Medical Center (MC), Bronx, NY

Nested variant of urothelial carcinoma is characterized by confluent small nests and abortive tubules of mildly atypical neoplastic cells infiltrating the lamina propria and/or muscularis propria of the bladder. Despite its deceptively bland histomorphologic appearance, the lesion is reported to have an aggressive behavior. The collective immunohistochemical expression of suppressor genes, growth factor, and proliferation activity marker has not been previously studied in this disease. Formalinfixed, paraffin-embedded archival tissues from 12 cases of nested variant of urothelial carcinoma were stained with monoclonal antibodies to p21, p27, p53, EGF-R, and bcl-2, as well as the proliferation marker MIB-1. The area of predominant immunoreactivity was also evaluated. The pattern of immunostaining was compared with the clinical parameters. $p 21$ was positive in 10 of 12 cases and located at the deepest portion of the tumor in 5 of 10 positive cases. Immunoreactivity for p27 was seen in 11 of 12 cases and limited to the superficial portion of the tumor in 9 of 11 positive cases. Only 3 and 2 of 12 cases were positive for $\mathrm{p} 53$ and bcl-2, respectively. MIB-1 immunoreactivity ranged from 2 to $35 \%$ of the neoplastic cells, with most tumors showing a proliferation index of $>15 \%$. Follow-up ranged from 3 to 30 months (mean, 17.6 mo). All patients except one were alive, although three patients developed metastases. Nested variant of urothelial carcinoma is a deceptively benign-appearing neoplasm with potential of deep invasion and metastases. Immunohistochemically, nested variant of urothelial carcinoma shares some features with

Copyright () 2003 by The United States and Canadian Academy of Pathology, Inc.

VOL. 16, NO. 12, P. 1289, 2003 Printed in the U.S.A.

Date of acceptance: July 18, 2003.

Address reprint requests to: Oscar Lin, M.D., Department of Pathology,

H-614, Memorial Sloan-Kettering Cancer Center, 1275 York Avenue, New

York, NY 10021; fax: 212-639-6318; e-mail: lino@mskcc.org.

DOI: 10.1097/01.MP.0000094091.04541.FC high-risk conventional urothelial carcinomas, such as loss of p27 expression and high proliferation index. Nevertheless, p53, bcl-2, or EGF-r immunoreactivity is not frequently seen.

KEY WORDS: Immunohistochemistry, Nested, Urothelial carcinoma.

Mod Pathol 2003;16(12):1289-1298

Nested variant of urothelial carcinoma was described as a malignant entity by Talbert and Young (1). This lesion has a deceptive benign appearance, characterized by confluent small nests or abortive tubules of urothelial cells infiltrating the lamina propria and/or bladder muscularis propria. They contain urothelial cells with mild pleomorphism, slightly increased nuclear/cytoplasmic ratio (N/C), and occasional prominent nucleoli (Fig. 1).

There are $<30$ cases of nested variant of urothelial carcinoma reported in the literature, suggesting that this lesion is either rare or underrecognized (1-8). The largest series (16 cases), was reported by Drew et al. (3), and the remaining reported cases originate from several small series and case reports. Nested variant of urothelial carcinoma is believed to have an aggressive behavior (3), despite its bland morphology, emphasizing the importance in recognizing this unusual variant of urothelial carcinoma.

Multiple biological markers have been evaluated in conventional urothelial carcinomas in an attempt to better understand the molecular events that lead to disease progression and study the correlation with grade and behavior. Some of these biological markers include p21, p27, p53, EGF-r, bcl-2, and MIB-1. In general, loss or absence of immunoreactivity for cyclin-dependent kinase inhibitors p21 and p27 has been associated with poor prognosis in conventional urothelial carcinoma (912). Overexpression of the tumor suppressor gene p53 has been related to tumorigenesis, prognosis, and survival in conventional urothelial carcinoma 

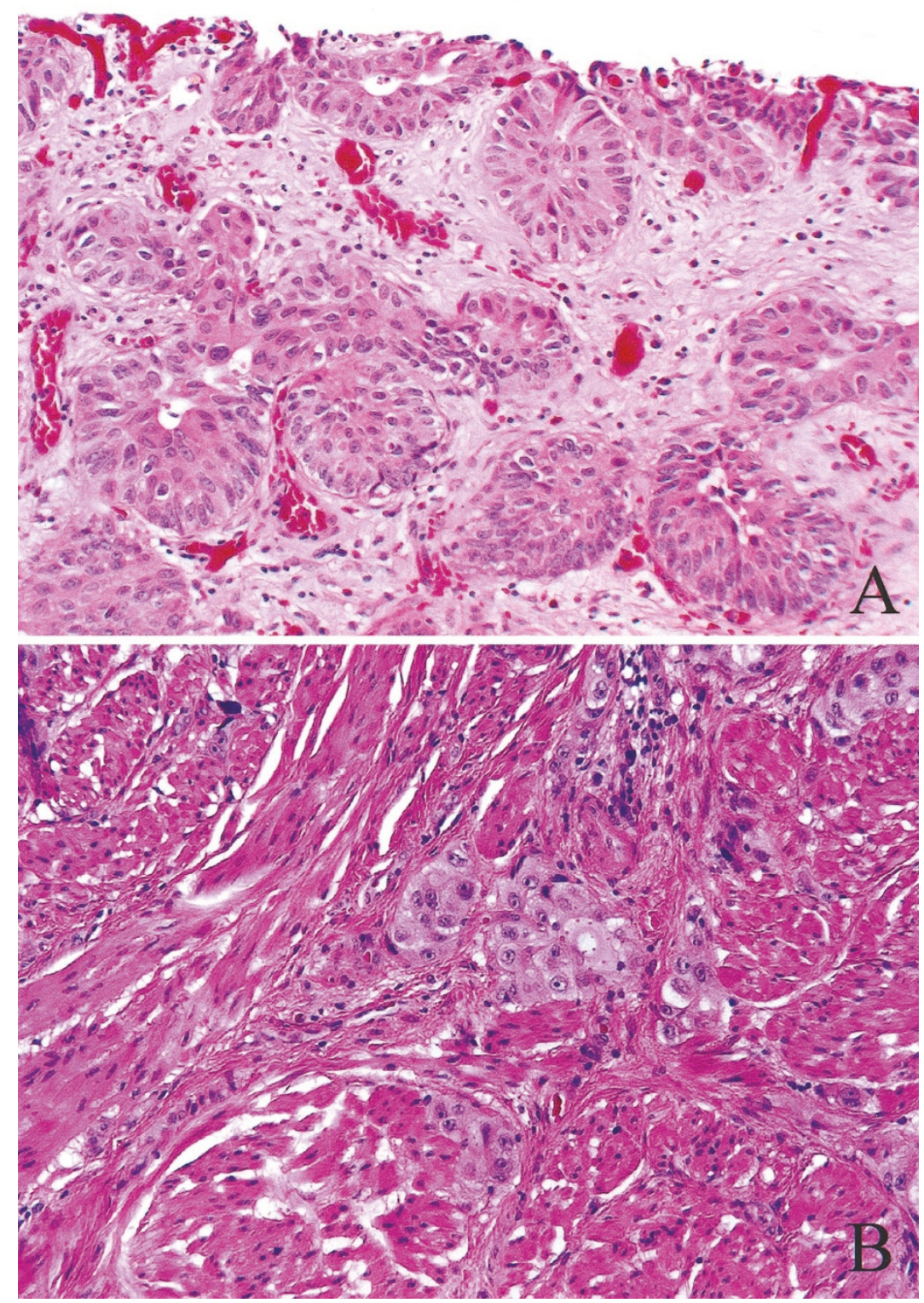

FIGURE 1. Nested variant of urothelial carcinoma is characterized by confluent small nests and abortive tubules of neoplastic cells infiltrating the lamina propria and/or bladder muscularis propria.

(11-17). Expression of the apoptosis-regulating protein bcl-2 and the receptor for the epidermal growth factor EGF-r have also been linked with poor prognosis and with high-stage conventional urothelial carcinoma $(15,16,18)$. Hence, to evaluate potential role of these biological markers in nested variant of urothelial carcinoma, we studied their expression in a series of 12 cases.

\section{MATERIALS AND METHODS}

Twelve cases of urothelial carcinoma with the morphologic characteristics of nested variant of urothelial carcinoma as described by Talbert and Young (1) were identified from the files of Memorial Sloan-Kettering Cancer Center. All patients were treated and followed at the same institution. The diagnosis of nested variant of urothelial carcinoma was reviewed and confirmed by three authors (OL, MC, and VER). The specimens were also evaluated for the presence of conventional urothelial carcinoma. The demographic information, clinical presentation, pathologic stage, and follow-up were extracted from the medical charts.

Tissue fixed in $10 \%$ formalin and embedded in paraffin was available in all cases. Immunohistochemical studies were performed using the avidinbiotin-peroxidase complex method. The primary antibodies used were p21 $1^{\mathrm{WAF} 1}$ (Oncogene, Cambridge; 1:100 dilution), p27 ${ }^{\mathrm{Kip} 1}$ (DAKO, Carpinteria, CA; 1:2000 dilution), p53 (Clone DO7, DAKO; 1:1000 dilution), MIB-1(Immunotech, Fullerton, CA; 1:50 dilution), bcl-2 (DAKO; 1:50 dilution), and EGF-r (Zymed, South San Francisco, CA; 1:100 dilution). 
Immunostaining enhancement with heat epitope retrieval procedure was performed for the antibodies p21 ${ }^{\mathrm{WAF} 1}, \mathrm{p} 27^{\mathrm{Kip} 1}$, p53, MIB-1, and bcl-2 using a regular vegetable steamer. Briefly, the sections were deparaffinized and placed in a solution of citrate buffer solution ( $\mathrm{pH}$ 6.0), steamed for 30 minutes, then cooled before immunostaining. Prior digestion with pepsin was used for EGF-r immunostaining. The antigen-antibody reaction was visualized using 3,3'-diaminobenzidine as chromogen. Known positive and negative tissues were used as controls. The thresholds that were used to call a lesion positive or negative were $5 \%$ of nuclear staining for $\mathrm{p} 21^{\mathrm{WAF} 1}$ and $\mathrm{p} 27^{\mathrm{Kip} 1}, 20 \%$ nuclear staining for $\mathrm{p} 53$, $5 \%$ cytoplasmic staining for bcl-2, and 5\% membranous staining for EGF-r. MIB-1 immunostaining was reported as percentage of nuclear staining. The distribution of immunoreactivity was also analyzed in addition to the presence or absence of immunoreactivity.

\section{RESULTS}

The clinicopathologic findings of the 12 patients with nested variant of urothelial carcinoma are summarized in Table 1 . The patients in this series included 9 male and 3 female patients ranging in age from 42 to 90 years old (mean $=65 \mathrm{y}$ ). Ten of 12 patients underwent cystectomy, 1 patient received only radiation therapy because of the presence of distant metastases, and 1 patient underwent transurethral resection only. All lesions were composed of small, well-delineated nests of urothelial cells infiltrating the lamina propria and/or bladder muscularis propria. Direct extension to the bladder mucosal surface was seen in 11 of 12 cases. The neoplastic cells displayed mild increase in N/C ratio, dense cytoplasm, and occasional nucleoli resulting in a bland morphology. Increased pleomorphism was seen occasionally in the deepest components of the tumor. Mitotic figures were not readily identified. Nested variant of urothelial carcinoma was the only type of urothelial carcinoma present in seven patients, and it was the only type seen in the invasive component in all specimens. The remaining five patients presented with overlying in situ conventional urothelial carcinoma. Four of the associated in situ carcinomas were flat, and one was a low-grade papillary carcinoma. No direct connection was seen between flat urothelial carcinoma in situ and the nested variant of urothelial carcinoma (Fig. 2A). The papillary carcinoma was present, juxtaposed to the nested variant of urothelial carcinoma (Fig. 2B). No other type of invasive urothelial carcinoma was seen in these 12 patients. Pathologic stage varied from pT1 to pT3b (3pT1, 5pT2, and 4pT3), clinical stage (AJCC 2002) ranged from I to IV, and the follow-up ranged from 3 to 30 months $($ mean $=17.6 \mathrm{mo})$. At time of last follow-up, nine patients had no evidence of disease, two patients were alive with metastases, and one patient had died of disease. The latter three patients represent patients who were clinically Stage IV at the time of diagnosis, with regional metastases at the time of cystectomy or presence of clinically evident distant metastases.

Two pathologists (OL and VER) analyzed the immunohistochemical findings independently, and the results represent a consensus of their findings. In general, there was excellent interobserver agreement. The immunohistochemical results and the distribution of immunoreactivity for each individual case are summarized in Table 2.

The majority of cases were positive for p21 and p27, whereas immunoreactivity for p53 was seen in only three cases. Two cases showed immunoreactivity for bcl-2 confined to the superficial component of the tumor. No immunoreactivity for EGF-r was seen in any of the cases. Proliferation activity using the MIB-1 immunoreactivity was present in all cases and, in most cases, with a proliferative index of $>15 \%$ (Table 2).

The distribution of immunoreactivity within tumors was interesting. p21 was located predominantly in the deepest component of the tumor in 5 of 10 p21-positive cases (Fig. 3A) and was diffusely

TABLE 1. Clinicopathologic Features

\begin{tabular}{|c|c|c|c|c|c|c|c|}
\hline Patient & Age/Sex & Pathologic Stage & AJCC Stage & Treatment & Status & Follow-Up & Associated CUC (WHO/ISUP 1998) \\
\hline 1 & $90 / \mathrm{F}$ & pT1 & I & Cystectomy & NED & 30 & No \\
\hline 2 & $73 / \mathrm{F}$ & pT3b & III & Cystectomy + chemotherapy & NED & 5 & No \\
\hline 3 & $65 / \mathrm{M}$ & pT2b & IV & Cystectomy + chemotherapy & AWM & 16 & Flat CIS \\
\hline 4 & $70 / \mathrm{M}$ & pT3b & III & Cystectomy + chemotherapy & AWM & 15 & Flat CIS \\
\hline 5 & $61 / \mathrm{M}$ & pT3b & IV & Cystectomy + chemotherapy & NED & 19 & Flat CIS \\
\hline 6 & $80 / \mathrm{M}$ & pT3b & III & Cystectomy & NED & 27 & Flat CIS \\
\hline 7 & $66 / \mathrm{M}$ & pT1 & I & Cystectomy & NED & 22 & No \\
\hline 8 & $53 / \mathrm{M}$ & pT1 & I & Cystectomy & NED & 19 & No \\
\hline 9 & $51 / \mathrm{M}$ & pT2 & IV & RT & DOD & 3 & No \\
\hline 10 & $59 / \mathrm{M}$ & pT2a & II & Cystectomy & NED & 24 & No \\
\hline 11 & $42 / \mathrm{F}$ & pT2a & II & Cystectomy & NED & 20 & No \\
\hline 12 & $76 / \mathrm{M}$ & pT2a & I & TUR & NED & 12 & Low Grade Papillary UC \\
\hline
\end{tabular}

M, male; F, female; NED, alive with no evidence of disease; AWM, alive with metastases; DOD, dead of disease; CIS, carcinoma in situ; CUC, conventional urothelial carcinoma; TUR, transurethral resection. 


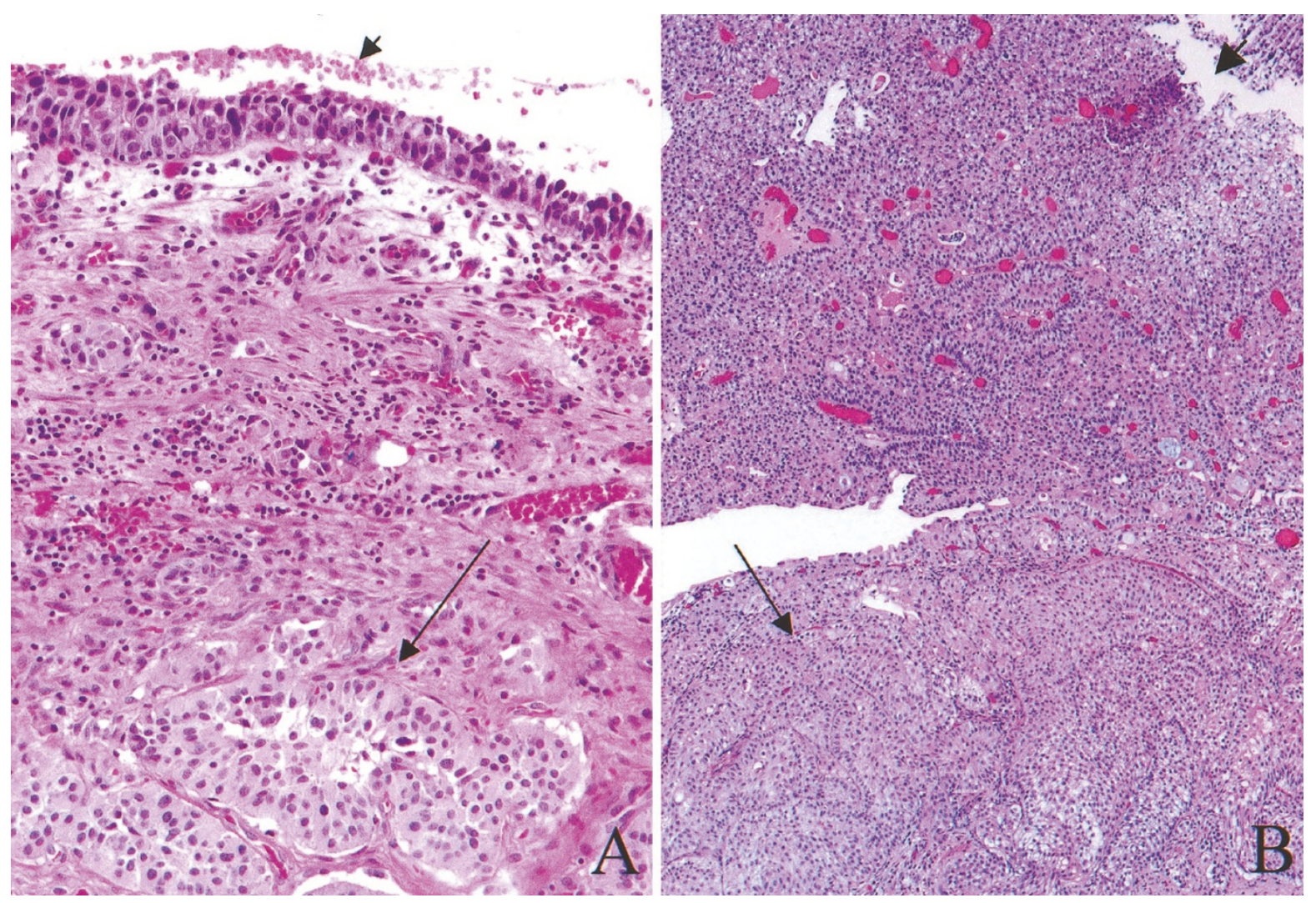

FIGURE 2. A, no direct connection was seen between overlying flat urothelial carcinoma in situ (short arrow) and the nested variant of urothelial carcinoma (long arrow). B, low-grade papillary carcinoma (short arrow) was present, juxtaposed with the nested variant of urothelial carcinoma (long arrow).

TABLE 2. Immunohistochemical Results and Geographic Distribution of Several Markers in Nested Variant of Urothelial Carcinoma

\begin{tabular}{|c|c|c|c|c|c|c|c|c|c|c|c|}
\hline \multirow{2}{*}{ Case } & \multicolumn{2}{|c|}{$\mathrm{p} 21$} & \multicolumn{2}{|c|}{$\mathrm{p} 27$} & \multicolumn{2}{|c|}{ p53 } & \multirow{2}{*}{$\frac{\text { EGF-R }}{\text { Result }}$} & \multicolumn{2}{|c|}{ bcl2 } & \multicolumn{2}{|c|}{ MIB-1 } \\
\hline & Result & Location & Result & Location & Result & Location & & Result & Location & $\%$ & Location \\
\hline 1 & + & Diffuse & + & Surface & - & & - & - & & 20 & Diffuse \\
\hline 2 & + & Diffuse & + & Diffuse & - & & - & - & & 15 & Diffuse \\
\hline 3 & + & Diffuse & - & & - & & - & - & & 35 & Diffuse \\
\hline 4 & + & Diffuse & + & Surface & - & & - & - & & 35 & Diffuse \\
\hline 5 & + & Base & + & Surface & + & Base & - & + & Surface & 2 & Base \\
\hline 6 & - & & + & Surface & - & & - & - & & 20 & Diffuse \\
\hline 7 & + & Base & + & Surface & - & & - & + & Surface & 35 & Diffuse \\
\hline 8 & + & Base & + & Surface & - & & - & - & & 15 & Base \\
\hline 9 & - & & + & Surface & + & Diffuse & - & - & & 30 & Diffuse \\
\hline 10 & + & Diffuse & + & Surface & - & & - & - & & 30 & Base \\
\hline 11 & + & Base & + & Surface & - & & - & - & & 20 & Diffuse \\
\hline 12 & + & Base & + & Surface & + & Base & - & - & & 15 & Base \\
\hline
\end{tabular}

distributed in the remaining 5 cases (Fig. 3B). Immunohistochemical staining for p27 was seen in 11 cases. The immunoreactivity was localized only in the superficial component of the tumor in 10 of these 11 cases (Fig. 4A), whereas only one p27positive case displayed a diffuse immunoreactivity (Fig. 4B). Interestingly, five cases with p21 expression confined to the deepest component of the tumor showed p27 immunoreactivity only in the superficial component of the tumor. Overexpression of p53 was observed only in three cases. Predominant p53 overexpression was noted in the deepest areas of the tumor in two cases (Fig. 5A) and was diffusely distributed in the remaining case (Fig. 5B). No case showed immunoreactivity for EGF-r. Immunoreactivity for bcl-2 was seen in the superficial component of the tumor in two cases (Fig. 6). All cases showed immunoreactivity for MIB-1, ranging from 15-35\%, except for one case, in which only $2 \%$ of the cells were positive. Three cases showed increased immunoreactivity in the deepest component of the tumor (Fig. 7A), whereas the remaining nine cases displayed diffuse immunoreactivity (Fig. 7B). 


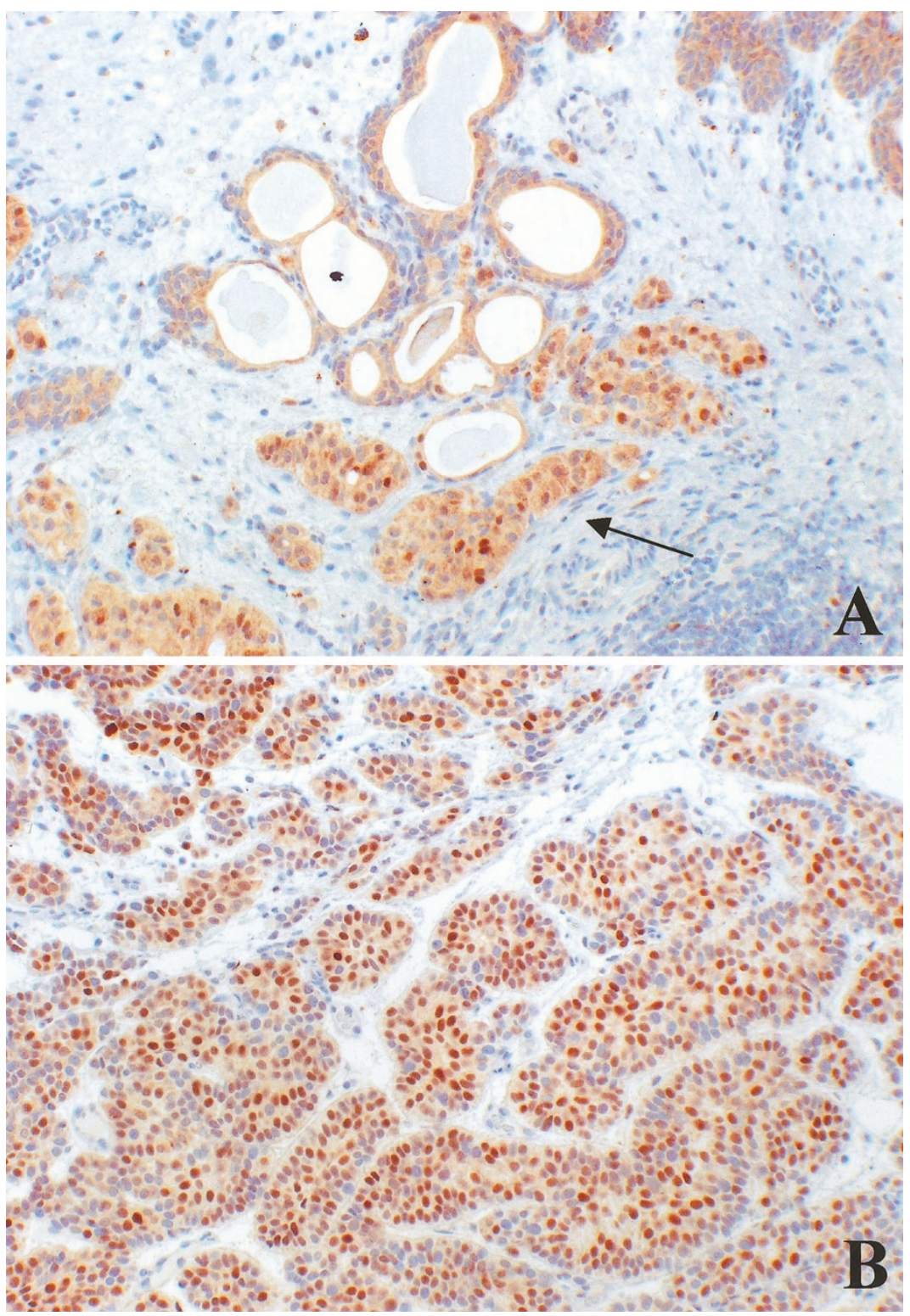

FIGURE 3. p21. A, immunostaining only in the deep component of the tumor (arrow). B, diffuse immunostaining.

\section{DISCUSSION}

Most carcinomas of the urinary bladder, flat or papillary, do not represent a major diagnostic problem for the pathologist. Nonetheless, some variants of urothelial carcinoma such as the nested variant have a bland morphology (19) and have growth pattern characteristics that overlap with various benign entities such as in nested papilloma, Brunn nests, and nephrogenic metaplasia. For these reasons, they might pose a diagnostic dilemma. The neoplastic cells in nested variant of urothelial carcinoma show little atypia, and they are arranged in nests that mimic cystitis cystica or Brunn's nests, leading to a possible underrecognition of this tumor. This lesion was even described as a benign lesion (20) before the original description of it as a malignant tumor by Talbert (1).
The demographics of our patient population are very similar to previously reported studies of nested variant of urothelial carcinoma. There is a marked predominance of male patients, with a mean age of $>60$ years. This age and sex distribution is similar to that in conventional urothelial carcinoma, a finding already noted by Drew et al. (3). The patients in our series presented with pathologic stage ranging from pT1 to pT3b ( 3 pT1, 5 pT2, and 4 pT3), whereas most patients previously described were reported as pT1 or pT2 (3). The higher stage presentation in our series might reflect the nature of our institution. Most patients (9 of 12) in our series were alive without evidence of disease at last follow-up, despite the higher stage presentation. Conversely, Drew et al. (3) reported a high incidence of persistent or progressive disease in his series. It is impor- 


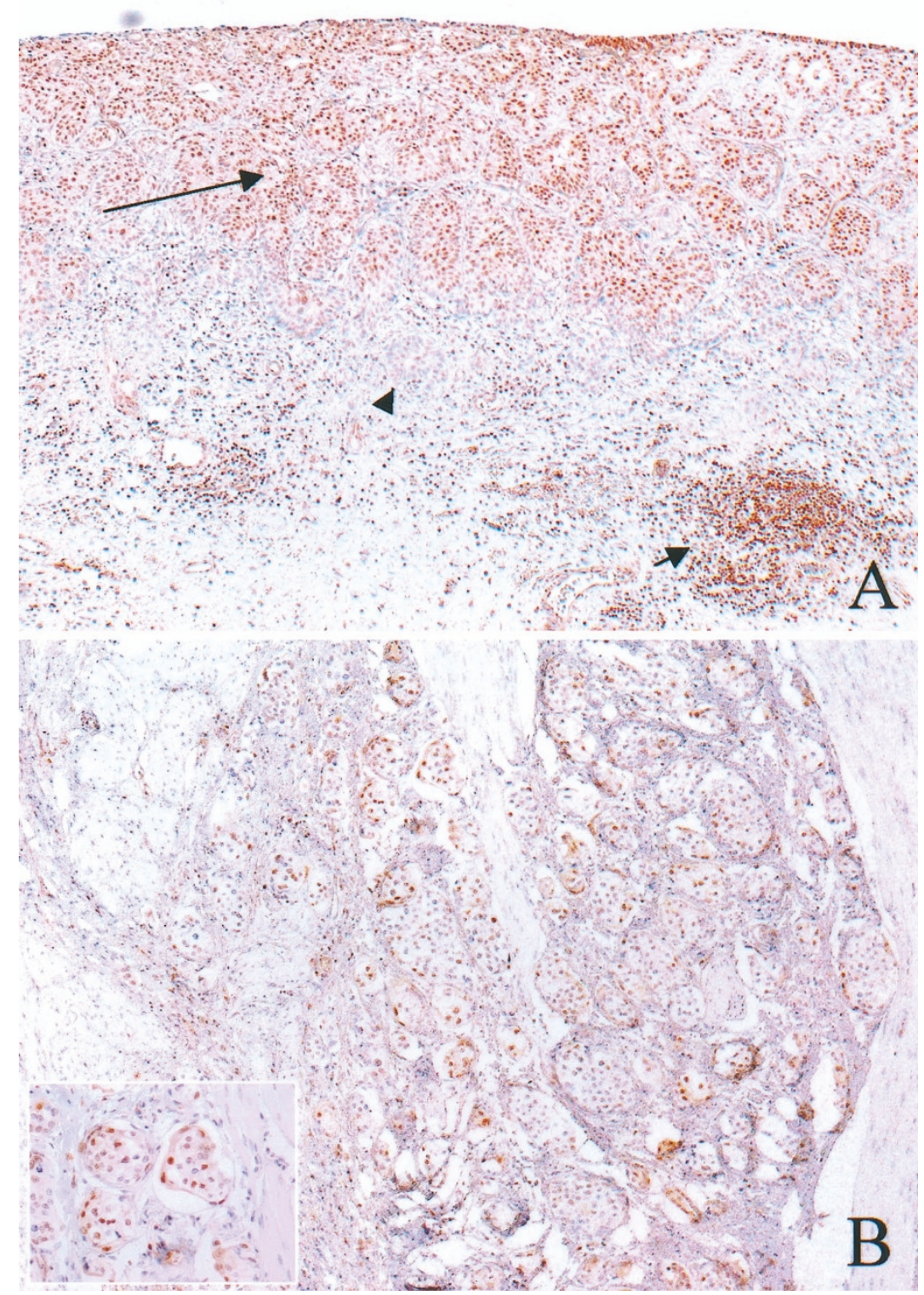

FIGURE 4. p27. A, presence of immunoreactivity in the superficial component only (long arrow) with loss of immunostaining in the deep component (arrowhead); note presence of reactivity in the lymphocytes (short arrow). B, diffuse immunostaining.

tant to indicate that the majority patients in our series were treated with cystectomy, whereas all cases reported by Drew et al. (3) were treated only with transurethral resection. These findings suggest that this disease should be approached clinically as high-risk disease, with early cystectomy as an option for pT1 and pT2 tumors. We have previously demonstrated that cytologic follow-up in these patients after TUR is difficult (21).

The aggressive behavior of nested variant of urothelial carcinoma underscores the importance of distinguishing them from benign proliferative lesions such as Brunn's nests, cystitis glandularis, nephrogenic metaplasia, and inverted papilloma. Cytologic atypia is not a good parameter because the mild atypia seen in nested variant of urothelial carcinoma can be deceptive, especially at low- and medium-power magnification. Obviously, invasion of the muscularis propria excludes the possibility of any benign lesion.

Previous studies performed in conventional urothelial carcinoma have indicated that alterations in cell-cycle regulation are important in the neoplastic transformation and progression (13). Some of the markers implicated in this process include p21, p27, p53, and bcl-2.

The cyclin-dependent kinase inhibitor p21 is a protein that can lead to cell cycle arrest at G1 phase by inhibiting DNA replication through interaction with PCNA (22). The presence of p21 expression has been associated with low-grade urothelial carcinomas (23) and decreased probability of tumor recurrence (2). Stein et al. (2) have demonstrated that the majority of tumors with overexpression of p53 


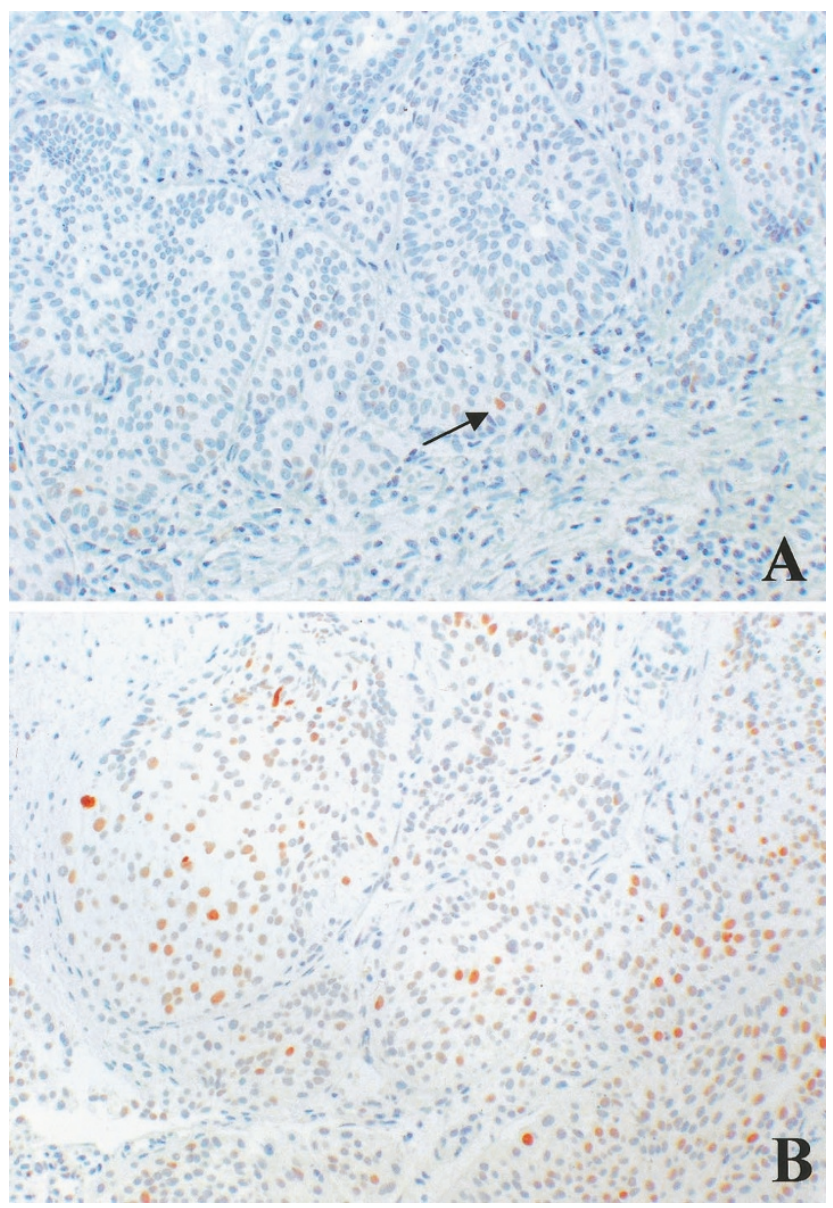

FIGURE 5. p53. A, rare immunoreactivity in the deep component of the tumor (arrow). B, diffuse immunostaining.

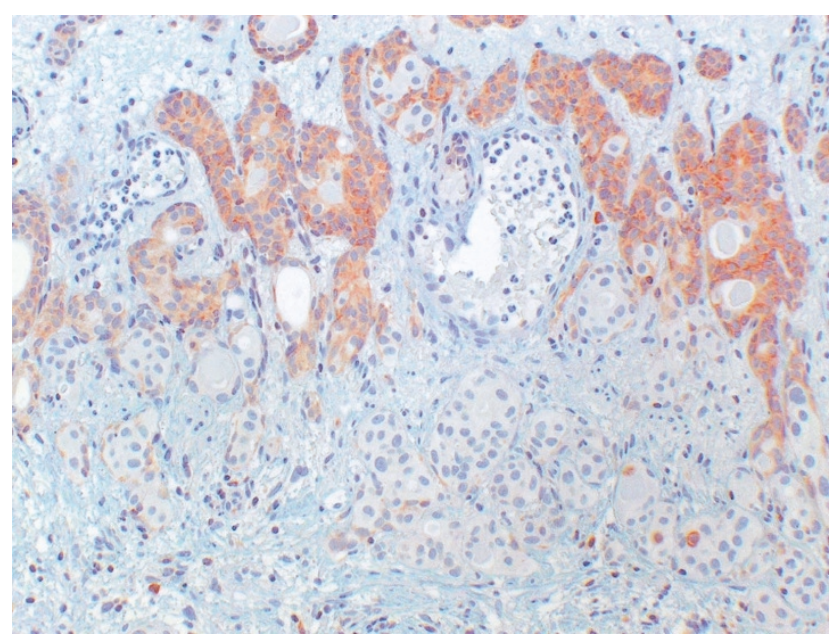

FIGURE 6. bcl-2. Immunostaining present only in the superficial component of the tumor.

showed loss of $\mathrm{p} 21$, whereas most tumors that are p21 positive contained wild-type p53. This is a finding similar to what was observed in our series. Eight of $10 \mathrm{p} 21$-positive cases showed no overexpression of p53, whereas 1 case with diffuse overexpression of p53 showed absence of expression of p21. The study by Stein et al. (2) also concluded that tumors with p53 overexpression and lack of p21 expression demonstrated higher rate of recurrence and worse survival when compared with p53 overexpression/ p21-positive and p53 wild type/p21-positive cases. This was also a finding of Qureshi et al. (11) in their study of nine cases of conventional urothelial carcinoma. Interestingly, the only patient who died of disease in our series displayed p53 overexpression and lack of p21 expression. We only encountered two cases with p53 overexpression in which p21 was expressed.

Most cases (10 of 12) showed expression of p21, with predominant expression in the deepest component of the tumor in 5 of 10 positive cases. Of note, increased proliferation activity as shown by MIB-1 immunoreactivity was also noted in the same area; therefore, the presence of p21 expression in the deepest component of tumor might indicate increased transcription of the protein in an attempt to stop the cell cycle by counterbalancing other tumorigenic pathways.

Other biological markers that have been studied in conventional urothelial carcinoma include p27, a p53-independent cyclin-dependent kinase inhibitor. Low p27 expression has been associated with progression of disease (9), poor overall survival, and relapse (10). In our series, low or absent p27 expression in the deeper component of the tumor in association with high proliferation index $(>15 \%)$, was seen in nine cases. These findings might suggest that loss of p27 and high proliferation index represent common tumorigenic pathways in nested variant of urothelial carcinoma.

The immunoreactivity of p27 in 10 of 11 p27 positive nested variant of urothelial carcinoma cases was quite peculiar because it was limited to the superficial component of the tumor. Furthermore, 5 of these 10 cases demonstrated immunoreactivity for $\mathrm{p} 21$ only in the deepest component of the tumor. This finding might suggest that, in selected cases, once p27 protein transcription is decreased, a different pathway induces the expression of p21 in an attempt to inhibit cell proliferation.

The widely studied tumor suppressor protein p53 has been associated with aggressive biological behavior in conventional urothelial carcinoma (24). However, its role as an independent prognostic factor in immunohistochemical studies has been disputed in the literature. This controversy may be attributed to lack of standards in performing and analyzing the immunohistochemical data. Nonetheless, p53 overexpression was not found to be a frequent event in nested variant of urothelial carcinoma.

Proliferation activity as seen by immunoreactivity for the proliferation marker Ki-67 using the antibody MIB-1 has been associated with aggressive- 


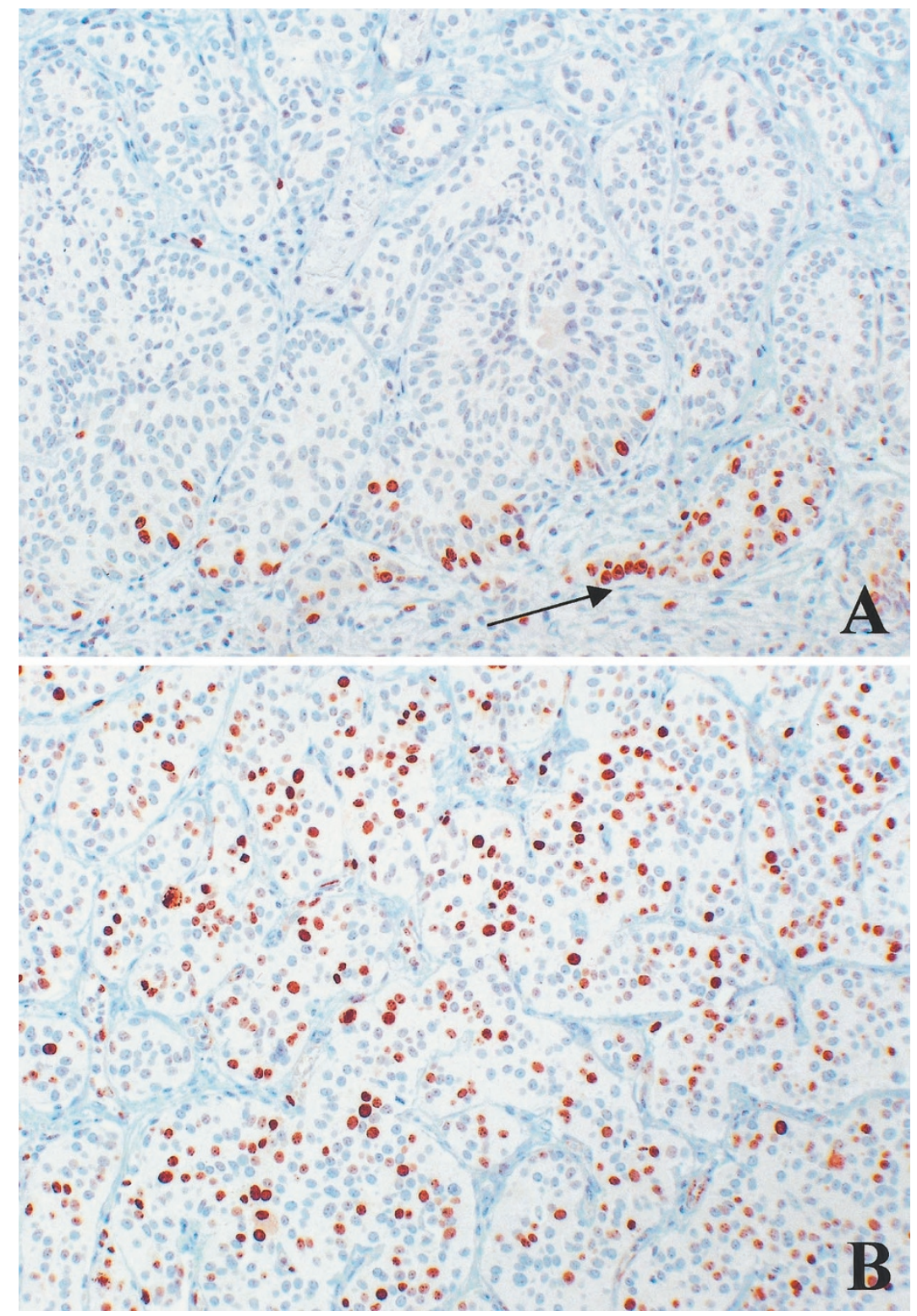

FIGURE 7. MIB-1. A, immunoreactivity limited to the deep component of the tumor (arrow). B, diffuse immunostaining.

ness in conventional urothelial carcinoma. Several investigators $(25,26)$ have indicated that proliferation activity is an independent indicator on univariate analysis. The relationship of proliferation activity with other biological markers such as p53 and p27 has also been well studied and appears to correlate with poor prognosis $(10,11,15)$. High proliferation index was frequently seen in nested variant of urothelial carcinoma and may be helpful in distinguishing nested variant of urothelial carcinoma from benign proliferative lesions such as cystitis cystica, Brunn's nests, nephrogenic adenomas, or inverted papillomas. These proliferative lesions are known to have a proliferation index that does not exceed $10 \%$, as opposed to nested variant of urothelial carcinoma (27-30). This feature can be useful in superficial biopsies in which the infiltrative nature of nested variant of urothelial carcinoma cannot be easily evaluated.

The apoptosis regulating protein bcl-2 is reported to correlate with poor patient outcome, particularly when associated with p53 overexpression $(15,16)$. This tumorigenic pathway does not appear to play a role as a prognostic factor in nested variant of urothelial carcinoma because expression of bcl-2 and p53 overexpression were not common events in our series. Only 1 case in our series showed bcl-2 expression in combination with p53 overexpression.

EGF-r is another biological marker that has been studied in conventional urothelial carcinoma and has been associated with poor prognosis and high proliferation index $(18,31)$. The expression of EGF-r also does not appear to play a role in nested variant of urothelial carcinoma because EGF-r expression 
was not seen in any case in our series, despite the high proliferation index seen in most cases.

The heterogeneous geographic distribution of biological markers in nested variant of urothelial carcinoma is interesting. It suggests that these tumors are composed of neoplastic cells with heterogenous phenotypes and that this heterogeneity can be geographically related. Intratumoral histologic, cytogenetic, and immunohistochemical heterogeneity is a known phenomenon in conventional urothelial carcinoma. It is not unusual to see the presence of glandular or squamous differentiation in close association with conventional urothelial carcinoma. Morphologic heterogeneity can also occur in nested variant of urothelial carcinoma, where increased atypia in the deepest component of the tumor has been described in the literature and it was present in one case in our series. Intratumoral cytogenetic heterogeneity has also been demonstrated in conventional urothelial carcinoma by comparative genomic hybridization (32), loss of heterozygosity, and single nucleotide polymorphism (33) studies. Diaz-Cano et al. (33) have also reported differences, although not significant, in immunohistochemical expression of p53, p21, and pRB in superficial and deepest component of muscle-invasive conventional urothelial carcinoma. Curiously, those investigators reported that p21 expression was higher in the superficial compartment of conventional urothelial carcinoma, whereas in our study, p21 was present diffusely or predominantly in the deepest component of nested variant of urothelial carcinoma. The presence of immunoreactivity only in the superficial component raised the possibility that fixation could be responsible for the difference in immunoexpression between different compartments. This problem was not an issue in our series because there was immunoreactivity in both superficial and deep compartments for different antibodies with equal intensity. Furthermore, immunoreactivity for p27 was seen in lymphocytes located deep in the tissue fragments, whereas p27 expression was seen only in the superficial component.

In summary, nested variant of urothelial carcinoma is a deceptively bland-appearing neoplasm with potential of deep invasion and metastases that should not go unrecognized. Immunohistochemically, nested variant of urothelial carcinoma shares some features with poor-prognosis conventional urothelial carcinoma, such as loss of p27 expression in the deeper component of the tumor and high proliferation index. Other biological markers associated with poor prognosis in conventional urothelial carcinoma, such as p53, bcl-2, and EGF-r, are not frequently altered in nested variant of urothelial carcinoma. Increased immunostaining for MIB-1 has the potential to become a helpful marker to distinguish nested variant of urothelial carcinoma from benign proliferative lesions, which it mimics.

\section{REFERENCES}

1. Talbert ML, Young RH. Carcinomas of the urinary bladder with deceptively benign-appearing foci. A report of three cases. Am J Surg Pathol 1989;13:374-81.

2. Stein JP, Ginsberg DA, Grossfeld GD, Chatterjee SJ, Esrig D, Dickinson MG, et al. Effect of p21WAF1/CIP1 expression on tumor progression in bladder cancer. J Natl Cancer Inst 1998;90:1072-9.

3. Drew PA, Furman J, Civantos F, Murphy WM. The nested variant of transitional cell carcinoma: an aggressive neoplasm with innocuous histology. Mod Pathol 1996;9:989-94.

4. Young RH, Oliva E. Transitional cell carcinomas of the urinary bladder that may be underdiagnosed. A report of four invasive cases exemplifying the homology between neoplastic and non-neoplastic transitional cell lesions. Am J Surg Pathol 1996;20:1448-54.

5. Murphy WM, Deana DG. The nested variant of transitional cell carcinoma: a neoplasm resembling proliferation of Brunn's nests. Mod Pathol 1992;5:240-3.

6. Tatsura H, Ogawa K, Sakata T, Okamura T. A nested variant of transitional cell carcinoma of the urinary bladder: a case report. Jpn J Clin Oncol 2001;31:287-9.

7. Ozdemir BH, Ozdemir G, Sertcelik A. The nested variant of the transitional cell bladder carcinoma: a case report and review of the literature. Int Urol Nephrol 2000;32:257-8.

8. Paik SS, Park MH. The nested variant of transitional cell carcinoma of the urinary bladder. Br J Urol 1996;78:793-4.

9. Kamai T, Takagi K, Asami H, Ito Y, Oshima H, Yoshida KI. Decreasing of p27(Kip1)and cyclin E protein levels is associated with progression from superficial into invasive bladder cancer. Br J Cancer 2001;84:1242-51.

10. Korkolopoulou P, Christodoulou P, Konstantinidou AE, Thomas-Tsagli E, Kapralos P, Davaris P. Cell cycle regulators in bladder cancer: a multivariate survival study with emphasis on p27Kip1. Hum Pathol 2000;31:751-60.

11. Qureshi KN, Griffiths TR, Robinson MC, Marsh C, Roberts JT, Lunec J, et al. Combined p21WAF1/CIP1 and p53 overexpression predict improved survival in muscle-invasive bladder cancer treated by radical radiotherapy. Int J Radiat Oncol Biol Phys 2001;51:1234-40.

12. Pfister C, Larue H, Moore L, Lacombe L, Veilleux C, Tetu B, et al. Tumorigenic pathways in low-stage bladder cancer based on p53, MDM2 and p21 phenotypes. Int J Cancer 2000;89:100-4.

13. Cordon-Cardo C. Mutations of cell cycle regulators. Biological and clinical implications for human neoplasia. Am J Pathol 1995;147:545-60.

14. Baithun SI, Naase M, Blanes A, Diaz-Cano SJ. Molecular and kinetic features of transitional cell carcinomas of the bladder: biological and clinical implications. Virchows Arch 2001; 438:289-97.

15. Wolf HK, Stober C, Hohenfellner R, Leissner J. Prognostic value of p53, p21/WAF1, Bcl-2, Bax, Bak and Ki-67 immunoreactivity in pT1 G3 urothelial bladder carcinomas. Tumour Biol 2001;22:328-36.

16. Ong F, Moonen LM, Gallee MP, ten Bosch C, Zerp SF, Hart AA, et al. Prognostic factors in transitional cell cancer of the bladder: an emerging role for Bcl-2 and p53. Radiother Oncol 2001;61:169-75.

17. Pfister C, Moore L, Allard P, Larue H, Lacombe L, Tetu B, et al. Predictive value of cell cycle markers p53, MDM2, p21, and Ki-67 in superficial bladder tumor recurrence. Clin Cancer Res 1999;5:4079-84.

18. Sauter G, Haley J, Chew K, Kerschmann R, Moore D, Carroll $\mathrm{P}$, et al. Epidermal-growth-factor-receptor expression is as- 
sociated with rapid tumor proliferation in bladder cancer. Int J Cancer 1994;57:508-14.

19. Eble JN, Young RH. Carcinoma of the urinary bladder: a review of its diverse morphology. Semin Diagn Pathol 1997; 14:98-108.

20. Stern JB. Unusual benign bladder tumor of Brunn nest origin. Urology 1979;14:288-9.

21. Cardillo M, Reuter VE, Lin O. Cytologic features of the nested variant of urothelial carcinoma: a study of seven cases. Cancer 2003;99:23-7.

22. Waga S, Hannon GJ, Beach D, Stillman B. The p21 inhibitor of cyclin-dependent kinases controls DNA replication by interaction with PCNA. Nature 1994;369:574-8.

23. Clasen S, Schulz WA, Gerharz CD, Grimm MO, Christoph F, Schmitz-Drager BJ. Frequent and heterogeneous expression of cyclin-dependent kinase inhibitor WAF1/p21 protein and mRNA in urothelial carcinoma. Br J Cancer 1998;77:515-21.

24. Sarkis AS, Dalbagni G, Cordon-Cardo C, Zhang ZF, Sheinfeld J, Fair WR, et al. Nuclear overexpression of p53 protein in transitional cell bladder carcinoma: a marker for disease progression. J Natl Cancer Inst 1993;85:53-9.

25. Popov Z, Hoznek A, Colombel M, Bastuji-Garin S, LefrereBelda MA, Bellot J, et al. The prognostic value of p53 nuclear overexpression and MIB-1 as a proliferative marker in transitional cell carcinoma of the bladder. Cancer 1997;80:147281.

26. Asakura T, Takano Y, Iki M, Suwa Y, Noguchi S, Kubota Y, et al. Prognostic value of Ki-67 for recurrence and progression of superficial bladder cancer. J Urol 1997;158:385-8.
27. Limas C. Proliferative state of the urothelium with benign and atypical changes. Correlation with transferrin and epidermal growth factor receptors and blood group antigens. J Pathol 1993;171:39-47.

28. Isimbaldi G, Di Nuovo F, Sironi M, Catania G, Bonacina R, Privitera $\mathrm{O}$, et al. [Nephrogenic adenoma of the bladder. Morphological and immunophenotypic study with particular attention to differential diagnosis]. Pathologica 1999;91: $192-7$.

29. Pycha A, Mian C, Reiter WJ, Brossner C, Haitel A, Wiener H, et al. Nephrogenic adenoma in renal transplant recipients: $\mathrm{a}$ truly benign lesion? Urology 1998;52:756-61.

30. Valero Puerta JA, Redondo Martinez E, Jiemenez Gonzalez C, Gomez Medina ML, Martinez de la Riva SI. [Inverted transitional-cell papilloma: the expression of the Ki-67 nuclear antigen as a prognostic factor]. Arch Esp Urol 1995;48: 887-92.

31. Messing EM. Clinical implications of the expression of epidermal growth factor receptors in human transitional cell carcinoma. Cancer Res 1990;50:2530-7.

32. Voorter C, Joos S, Bringuier PP, Vallinga M, Poddighe P, Schalken J, et al. Detection of chromosomal imbalances in transitional cell carcinoma of the bladder by comparative genomic hybridization. Am J Pathol 1995;146:1341-54.

33. Diaz-Cano SJ, Blanes A, Rubio J, Matilla A, Wolfe HJ. Molecular evolution and intratumor heterogeneity by topographic compartments in muscle-invasive transitional cell carcinoma of the urinary bladder. Lab Invest 2000;80:279-89. 